\title{
Política, economía y fiscalización de un espacio urbano. El establecimiento de la Aljama de los judíos en la Mallorca cristiana ${ }^{1}$
}

\author{
JoRge MAiz CHACÓN ${ }^{2}$ \\ UNED (C.A. ILLES BALEARS)
}

«E cels qui s'eren reculits e. un castell dels jueus, reteren-se al rey per fer ses volentats»

[Bernat Desclot: Crònica]

\begin{abstract}
RESUMEN
En el texto analizamos la llegada de los judios a Mallorca después de 1229. Se abordan diversas problemáticas como su instalación inicial cerca de la Almudayna, actividades que desarrollan y las distintas

politicas de los monarcas (Cartas de

Franqueza, Orden de los Templarios)

hasta la creación de la aljama balear.

Analizamos nuevas fuentes sobre las

operaciones de compra y venta entre judios y cristianos momentos antes de la creación del nuevo barrio judio.
\end{abstract}

\section{PALABRAS CLAVE}

Historia de Mallorca, 1229-1312, Judios, Aljama, Templarios, Economía.

\section{ABSTRACT}

In the text we analyzed the arrival from the Jews to Majorca after 1229. They are approached diverse problematic like its initial installation near the Almudayna, activities that they develop and the different policies from the monarchs (Cartas de Franqueza, Orden de los Templarios) until the creation of the Balearic aljama. We analyzed new sources on the operations of purchase and sale between Jews and Christians moments before the creation of the new Jew district.

KEY WORDS

Historv of Majorca, 1229-1312, Jews,

Aljama, Templars, Economy.

1 Este trabajo forma parte del proyecto «Sociedad y organización fiscal: los impuestos indirectos en el remo de Mallorca (siglos XIV-XVI)". [BHA2003-04785] financiado por el Ministerio de Ciencia y Tecnologia.

2 http://www.medievalismo.org

jmaiz@palma.uned.es 


\section{EL PROCESO DE CONQUISTA Y LA DINAMIZACIÓN ECONÓMICA DEL TERRITORIO BALEAR}

La fecha de 1229, supone el inicio de una nueva etapa histórica para el espacio geográfico balear. Este mismo año, se inicia el proceso de conquista y cristianización de su territorio, así como su nueva articulación política, social, cultural y económica. Jaime I el Conquistador, decidirá la creación de un nuevo reino, con una ordenación jurídica y administrativa propia: el Reino de Mallorca. Fundamental para el estudio de su articulación es la Carta de Franqueza de $1230^{3}$, otorgada por el monarca a sus nuevos súbditos. Ésta, será la primera de las medidas encaminadas a la vertebración de la nueva empresa.

Tras las reformas derivadas de la Carta, se procede a la reordenación territorial del nuevo reino. Estas disposiciones, se hallan en el denominado Llibre del Repartiment ${ }^{4}$, cuyas consecuencias estarán también sujetas a otras medidas legales contemporáneas, que en suma sistematizarán la nueva situación política. Varias de las medidas, poseen la clara intencionalidad de facilitar el establecimiento de nueva población en Mallorca. Asi, el monarca - momentos después de la conquistaofrecerá una serie de pautas, entre ellas: exime durante diez años, el pago de cualquier tipo de contribución y servicio ${ }^{5}$, facilitando - en gran medida- de este modo el asentamiento de nuevos contingentes demográficos. Entre los nuevos y diversos grupos establecidos en el territorio balear ${ }^{6}$, encontramos algunas menciones al sujeto de este estudio: los judíos. Este grupo demográfico, social y religioso, procede en buena medida de otros territorios de la Corona de Aragón, de territorios francos, así como de pequeñas agrupaciones existentes años antes a la citada conquista. Para ello, contamos con el testimonio del Liber Maiolichinus, fuente que recoge los épicos y literarios avatares de la razzia pisano-catalana de 1113-1114, recogiendo las siguientes palabras: «El Comte de Barcelona, fent via per una altra part, rep en rendició tots els jueus" ${ }^{7}$, siendo muestra de la propia existencia de los mismos.

3 Santamaria Arandez, Álvaro: "La Carta de Franquesa de Maliorca, estatuto constituyente del Reino", Anuario de Estudios Medievales [Barcelona], 17 (1987), pp. 207-228. Cateura Bennasser, Pau: "Ampliación de la corona de Aragón. 1: El Reino de Mallorca", La Corona de Aragón. IV, La Consolidación, Aragó. Zaragoza, 1988, pp. 102-171.

4 Soto Company, Ricard: El còdex català del Llibre del Repartiment de Mallorca, Govern Balear 1984, Palma.

5 Pérez Martinez, Lorenzo: "Corpus documental balear: reinado de Jaime I", Fontes Rerum Balearium [Palma], I (1977), p. 84, documento 7 .

${ }^{5}$ Junto con los contigentes de grupos catalanes, las comunidades Marsella y de Montpellier aportan un importante número de nuevos moradores. Para analizar su trascendencia: Santamaria Arandez, Álvaro: "El patrimonio de las comunidades de Marsella y Montpellier en el Repartimiento de Mallorca". Actes du XII Congrés d'Histoire de la Couronne d'Aragon 'Montpellier. la Couronne d'Aragon et les Pays de Langue d'Oc (1204-1349), I, Montpellier, 1987, pp. 105-133.

7 Liber maiolichinus de gestis pisanorum illustribus, Traducción de Mireia Mulet Más, Societat Arqueològica Lul liana, Palma, 1991. p. 66. Este pasaje hace referencia a la rendición de los judíos que bajo dominación musulmana habitan sus tierras, y por tanto a su propia presencia, aunque del mismo testimonio no podemos extraer mucha más información. 
La historia judia, y en especial, los judios mallorquines en la Edad Media, han sido durante varios siglos, objeto de análisis y detenimiento; pero, si nos atenemos a la bibliografía aparecida ${ }^{8}$, observamos con suma preocupación que no existe ninguna monografía específica que haya tratado - de manera rigurosa - los aspectos cruciales para la agrupación de dicho grupo étnico y religioso en un Shekhunat o barrio específico con cierta autonomía propia. Es decir, ¿porqué en un determinado momento se decide concentrar a la minoria hebrea en un espacio geográfico?, ¿qué intencionalidad posee el mismo?, ¿cuáles son las consecuencias? Antes de discernir sobre estas preguntas, será necesario establecer los comportamientos cronológicos, económicos y territoriales en los momentos iniciales del proceso de llegada de estos nuevos y viejos pobladores.

Si nos detenemos en el reparto de la isla de Mallorca, una considerable parte, será de patrimonio real. La misma, a su vez, se redistribuye en tres importantes porciones; una primera al infante Pedro de Portugal, una segunda a las comunidades urbanas que participan en la conquista; y, en tercer lugar, la comunidad judia, a la que el 11 de julio de 1231, se le concede el estatuto jurídico y una dotación de bienes inmobiliarios rústicos y urbanos. Concediéndoles en la ciudad, propiedades en la zona de la Almudaina para la construcción de una sinagoga ${ }^{9}$, plaza, cementerio y casas. La localización del espacio judio, obedece a múltiples factores, pero su ubicación - al menos en los territorios de la antigua Corona de Aragón ${ }^{10}$ - suele emplazarse en torno al palacio, fortaleza o catedral. En el repartimiento de la Ciudad de Mallorca, Nuño Sans recibirá una importante dote inmobiliaria situada en zonas selectivas de la misma ${ }^{11}$, por tanto aquí las novedades serán mínimas. Así, encontramos una significativa porción en la zona marítima de la ciudad, en esta, se ubicarán progresivamente colonizadores-mercaderes genoveses, pisanos y judios principalmente. Es el propio Conde, quién cederá a determinados judios algunas de sus posesiones. Así, Gotzalbo Sastre ${ }^{12}$, Samuel ${ }^{13}$, Ceyt Abenceyt ${ }^{14}$, Juceff Almocatil ${ }^{15}$, Barobe de Alexandria ${ }^{16}$, Juceff de Massilia ${ }^{17}$ y As-

\footnotetext{
8 Véase nuestros trabajos sobre historiografía mallorquina: «Aproximación a la Mallorca bajomedieval. Producción historiográfica (1960-1998)". Medievalismo. Boletin de la Sociedad Española de Estudios Medievales [Madrid], 10 (2000), pp. 269-334; "La historiografía medieval mallorquina durante el franquismo (1939-1968)", III Simposio Internacional de Jóvenes Medievalistas, Lorca (en prensa).

Archivo Histórico Nacional, Pergaminos, Carp. 75, documento citado en: Pérez Martinez, Lorenzo: "Corpus docurnental...pp. 83-86

10 Assis, Yom Tov: The golden age of aragonese jewry. Community and Society in the Crown of Aragon, 1213-1327, The Littman Library of Jewish Civilisation, London-Oregon, 1997, p. 199.

11 Cateura Bennasser, Pau: «Repoblación, urbanización y comercio. El puerto de la Ciudad de Mallorca durante el siglo XIII", Mayurqa [Palma], 21 (19875-1987), pp. 91-99.

12 AGUILO, Estalisnao de K.: “Capbreu, ordenat l'any 1304, dels establiment y donacions ferts per Don Nuno Sans de cases y solars de la seua porció de la ciutat", Bolleti de la Societat Arqueológica Lul liana [Palma], XIV (1912-1913), p .210, doc. 13.

13 AGUILo, Estalisnao de K.: “Capbreu, ordenat l'any... p. 244, doc. 179, situada frente la Almudaina, en la misma ribera marítica

14 Aguito, Estalisnao de K.: “Capbreu, ordenat l'any... p. 244, doc. 181

15 Agullo. Estalisnao de K.: «Capbreu, ordenat l'any... p. 244, doc. 182; p. 251, doc. 241.

16) AGullo, Estalisnao de K.: "Capbreu, ordenat l'any... p. 245, doc. 195.

17 AGUILO, Estalisnao de K.: “Capbreu, ordenat l'any... p. 249, doc. 228.
} 
trugo Matza ${ }^{18}$ entre otros, se establecerán en su porción en el capbreu del año 1232/3; otros como Bonet ${ }^{19}$, accederán a éstas mediante compras. Al margen de esta porción, conservamos documentalmente la donación hecha por la aljama de judios al infante Pedro de Portugal de las casas que el rey Jaime I de Aragón, les había concedido en la zona de la Almudaina ${ }^{20}$.

De los contingentes cristianos que participan en el conflicto bélico, una aportación considerable podemos atribuirla a la Orden del Temple, que por la misma, recibirán 580 caballerías, hornos, donación de un castillo, y propiedades a extramuros de lo que hasta el momento era Madîna Mayûrqa. Esta importante aportación y posterior dote, poseerá algunas consecuencias como veremos posterioremente. Servir o prestar servicio regio, ofrecerá por tanto una serie de ventajas ${ }^{21}$, no solamente cuando se realiza repartos de tierras, o cuando quedan bajo el amparo de la protección real, sino que también llega a suponer una influencia en decisiones o el permiso de portar una vestimenta especial, etc. Aunque también puede significar, a medio y largo plazo, ser objeto de ciertas medidas o actuaciones perjudiciales.

Podemos afirmar que existe un política regia, encaminada a la potenciación del asentamiento de grupos - entre ellos judáicos - en Mallorca; la intencionalidad de estos primeros movimientos de carácter tolerante y de acogida, obedecen a varios aspectos:en un primer lugar, forman parte de la política repobladora de los nuevos territorios incorporados a la Corona de Aragón; del mismo modo, también son un trato de favor por la participación en el proceso preparativo (bien con préstamos $u$ otro tipo de ayudas), en el proceso de conquista y tratadística (básicamente en el trabajo de trajumanes) y en el posterior establecimiento de las normas política básica del nuevo territorio anexionado.

Los judíos, aunque reducidos en el porcentaje demográfico durante el siglo XIII, su importancia radicará en la activides que desempeñan, destacando en el acometido de actividades médicas, finanzas, comerciales y relacionadas con la administración. El monarca aragonés conocedor de algunos de sus favores, decide en las Cortes de Tarragona ${ }^{22}$ de 1235 , tomar medidas prohibitivas sobre los préstamos superiores al $20 \%$ de interés. Este ejercicio de cordura, podría estar sumamente relacionado con la petición que éste había realizado a acreedores, siendo excesiva anteriormente e intentando asi limitarla para futuras empresas.

18 Aguito, Estalisnao de K: "Capbreu, ordenat l'any... p. 250, doc. 234

19 Archivo Histórico Nacional, Clero, Cartujos, Carpeta 64, num. 3, 30 de enero de 1235, documento regestado en: Rossello Vaquer, Ramon: La Ciutat de Mallorca després de la conquista de 1229 (Documentari 1230-1300), Edicions Roig i Montserrat, Palma, 2004, p. 11.

20 Archivo del Reino de Mallorca, Pergaminos, Jaime I, num. 21, 6 abril 1236.

21 Romano Ventura, David: "Los hermanos Abenmenassé al servicio de Pedro el Grande de Aragón", Homenaje a Millás Vallicrosa, II, Consejo Superior de Investigaciones Científicas, Madrid, 1956, pp. 243-292; ver del mismo autor: "Courtisans juifs dans la Couronne d'Aragon", Les juits dans le Méditerranée médiévale et moderne, Université de Nice, Niza, 1986, pp. 79-95.

22 Cortes de los antiguos reinos de Aragón y Valencia y principado de Cataluña, Madrid, 1896-1907, citado en: Assis, Yom Tov: Jewish economy in the medieval Crown of Aragon. 1213-1327, Money and power, E. J. Brill, Brill's Series in Jewish Studies, XVIII, Leiden, 1997, p. 17 
Politica, economía y fiscalización de un espacio urbano. El establecimiento de la Aljama...

Tenemos atestiguada la actividad del judio Bahiel ${ }^{23}$ como trujamán del árabe en la conquista de Mallorca, quien aparece acompañando al conde Nuño. Esto supone, un trato de confianza para según que actividades, a la vez que el beneplácito y su posterior recompensa como ayudante de primer orden. Éstos, poseerán cierto privilegio, con la finalidad de reanudar ciertas empresas económicas, dinamizar el comercio y establecer, mediante ellos, ligazones "comerciales" con judíos del resto del Mediterráneo ${ }^{24}$.

Finalmente, no debemos olvidar, la necesidad de la corona de poseer una nueva base para poder potenciar el denominado sertor terciario, sabedores de la posibilidad que ciertos comerciantes-negociadores judios tienen con las redes internacionales de intercambio de mercancías. Así, Mallorca se adviene en un centro de redistribución de gran importancia dentro del mercado occidental mediterráneo. La importancia de determinados cortesanos judios, viene indicada por su presencia en los libros de repartimiento de Valencia, y en diversos pasajes de las crónicas medievales ${ }^{25}$, asi para Mallorca, en el Llibre dels Feyts concretamente Bahiel I, Salomó Alcostantini y Astruc Bonsenyor.

Los judíos de la Corona de Aragón, a diferencia de los del Norte del continente europeo, podían acceder a la tierra ${ }^{26}$, asi en este sentido, el Llibre del Repartiment anteriormente citado, hace claras evidencias de los territorios que reciben. Es importante, acceder al primer reparto del nuevo botín, ya que con la llegada de nuevos habitantes y la dinamización económica y comercial, estos territorios se revalorizan y adquieren una gran importancia. La colaboración de judios en la consquista, posee un claro y marcado carácter familiar, y concretado en el espacio en Aragón, Occitania, Castilla, Navarra, Sicilia o el Norte de África.

En el contexto expansionista de reinado de Jaime ${ }^{27}$, no debemos olvidar la necesaria penuria económica que supone emprender conflictos de carácter bélico, y el consecuente desarrollo fiscal que suele ir asociado al mismo. La necesidad de cierta liquidez, en territorios no aragoneses como por ejemplo Venecia ${ }^{28}$, se solucionará mediante el apremio que se ejerce en determinados momentos sobre la

23. La importancia de los ciertos judios como trajumanes ha sido analizada por David Romano, ofreciendo una visión de confianza real hacia determinados individuos o clanes. Ver: "Judíos escribanos y trujamanes del árabe en la Cororiá de Aragón (reinados de Jaime I a Jaime II), Sefarad [Madrid], XXXVIII (1978), pp. 71-105.

${ }^{24}$ Cateura Bennasser, Pau: "La contribución confesional: musulmanes y judios en el reino de MaHorca", Acta Historica et Archealogica Medieavalia [Barcelona], 20-21 (1999-2000), pp. 119-138.

2.5 Romano Ventura, David: "Les juifs de la Couronne d'Aragon dans les chroniques médiévales catalanes", XII Congrès d'Histoire de la Couronne d'Aragon, HI, Montpellier, 1988, pp. 25-31.

${ }^{26}$ Soto Company, Ricard: "Alguns homes rics: el paper dels jueus de Mallorca durant el segle XIIl», Butlleti de la Societat Catalana d'Estudis Histórics [Barcelona]. 7 (1996), pp. 27-39.

27 LALINDE ABADIA, Jesús: La Corona de Aragón en el Mediterráneo medieval (1229-1479), CSIC, Madrid, 1979.

${ }^{28}$ MuEller, Reinhold C.: "Les prêteurs juifs de Venise au Moyen Âge", Annales. Économies, Sociétés, Civilisations [Paris], 30.6 (1975), pp. 1277-1302. Existen otros estudios similares para el caso de Cerdeña, ver: DENJEAN, Claude: “Le crédit juif dans les campagnes cerdanes au XIIIe et XIVe siècle", Actes des XVIIes Journées Internationales d'Histoire de l'Abbaye de Flaran 'Endettement paysan et crédit rural dans l'Europe Medievale et Moderne', Presses Universitaires du Mirail, Toulouse, 1998, pp. 185-197. 
población judía. En algunas zonas castellanas ${ }^{29}$, también localizamos una importancia en el sistema hacendístico debido a la disponibilidad de capital. Estableciéndose así un movimiento bucle de presión-compensación entre ambas partes. Estos y otros movimientos, no siempre pueden ser analizados, sobretodo si nos atenemos a la dispersión y dificultad de las fuentes conservadas ${ }^{30}$. De entre las mismas, tenemos al ya citado Juceff Almocatil o Mocatil, será el encargado del pago de 1.124 sueldos y 9 dineros en $1246^{31}$, en concepto de los censales del año; también aparecerá en otras actividades político-económicas de cierta importancia; se encarga de la recaudación de censos reales para el patrimonio regio en $1269^{32}$. Las actividades económicas de este clan, no sólo podemos circunscribirlas a la capital del reino, puesto que también tenemos constancia de actividades realizadas en la part forana. Así en 1241, el propio Juceff Mocatil, adquiere del Monasterio de La Real ${ }^{33}$ todos las espletas ${ }^{34}$ del rafal Alpic y del término de Esporlas a cambio de 130 quarteras de vino. Por las mismas fechas ${ }^{35}$, continúan las actividades en la capital balear de nuevos individuos y grupos hebráicos de Marsella y otros lugares

En 1244, transcurridos ya más de diez años del proceso de conquista, se pone en marcha la contribución de la aljama judia a la hacienda regia, completándose la misma con el primer pago documentado: questia anual, o contribución, que sería regular y por una cantidad de 3.300 sueldos tres años más tarde ${ }^{36}$. Es durante gobierno del infante Jaime de Mallorca (1256-1276), cuando los judíos se encaminan según la historiografía tradicional, al menos dentro de la administración hacendistica en dos vertientes diferenciadas ${ }^{37}$ : como recaudadores y como arren-

29 Ortiz ReAL, Javier: Los judios de Cantabria en la Baja Edad Media, ADAL. Torrelavega, 1985. En su estudio, Javier Ortiz, evidencia como acaudillados judios gozarán de un trato diferenciado por parte de la Corona de Castilla, como compensación de determinadas cuantías económicas.

30 En este sentido, se pronunció hace tiempo Julio Valdeón, recogemos aquí sus palabras: "Las fuentes para el estudio de la Edad Media, ejercen en ocasiones, una verdadera dictadura para el investigador; limitando en muchas ocasiones el resultado de las mismas" [ver: "La Historia Social en España. Historia Medieval", La Historia Social en España. Actualidad y perspectivas, Editorial Siglo XXI, Madrid. 1991, pp. 149-163.].

31 Arxiu del Regne de Mallorca, Escrivania de Cartes Reials (a partir de ahora: ARM. ECR). 343, f. $100 \mathrm{v}$.

3: «judeo, collectori censualis domini Reig in Maioricis et patre suo Juceff Mocatil similiter tunc coHectori censulis predicti”, Archivo del Reino de Mallorca, Escrivania de Cartes Reials. 643, $f 105$ v. Este mismo cargo será ocupado por su hijo David Mocatil. Corresponde asimismo con la etapa 1265-1276. años en lo que se asigna una mayor influencia de los judios en la naciente administración mallorquina. Como ejemplo, realiza actividades - con permiso del lugarteniente real- de venta de esclavos sarracenos (ARM, ECR. 348, f. 45V) y obtiene de Pere de Caldes algunos censos y réditos durante un año (ARM, ECR. 347, f. 230)

3 Archivo del Reino de Mallorca, 342 Prot. Civil. Et Part. For. (1239-1261), 27 de agosto de 1241, documento citado en: Andrinal, L.; Mora, P.: Diplomatari del Monestir de Santa Maria de la Real de MaIlorca. I (1232-1360). Palma, 1982, doc. 23, p. 231

34 Segun la interpretación de Ricard Soto, se trata de una modalidad de derecho que consiste en la compra de una cosecha antes de que ésta se produzca. Ver: «La aljama judaica de Ciutat en el siglo XIII (época de Jaime I)", en Bolleti de la Societat Arqueológica Lul liana [Palma], XXXVI (1978), p. 165.

35 Vidal de Quilà, unas casas y corral en la ciudad (ARM, ECR 347, f. 16v, 25 de abril de 1268) y Bonisach de Marsella y Bonmacip de Marsella suplican la venta de unas casas de la mujer de Hug Gros por la deuda de diversos censos (ARM, ECR 347, f. 17, 12 de junio de 1268)

36 Cateura Bennasser, Pau: “La contribución confesional: musulmanes y judios...

3 Cateura Bennasser, Pau: «La contribución confesional: musulmanes y judios... 
Politica. economia y fiscalización de un espacio urbano. El establecimiento de la Aljama...

dadores. Asi, tienen a su cargo, el cobro de importantes rentas, como sería el caso de las rentas inmobiliarias de la Corona, los derechos reales sobre la Albufera de Alcudia, las rentas reales de granos de varias comarcas o el morabatín de sarracenos libres. La supuesta facilidad, con lo que los judios se hacen con estos derechos, nos hace reflexionar y acudir a la situación de llegada de estos nuevos habitantes. Las aljamas reales en la etapa preconquista, tenían la sufiente fuerza, como para aportar bien de manera voluntaria (a cambio de algo) o bien, de manera coercitiva, cantidades económicas a la empresa mallorquina, si ello sumamos los judios francos desplazados, nos encontramos con grupos de una ligazón e influencia tanto en lo económico como en lo político. El análisis realizado de los «instruments de franquesa», para los años 1263-1285, concentran las actividades de los mismos en una etapa cronológica muy concreta, los 1261-1269, éstas podría estar relacionadas con las nuevas necesidades financieras y fiscales del infante mallorquín, representando un $25,76 \%$ de la totalidad de las cartas ${ }^{38}$, del mismo modo que la nueva documentación analizada ofrece una nueva alza de las actividades de compra y venta para fines de la centuria (1291-1298) ${ }^{39}$.

Durante 1272, aparecen nuevas medidas correccionistas, que desembocan en la concesión de nuevos privilegios para los judios mallorquines. Éstos, ahora gozarán de las mismas prerrogativas que se han concedido a los barceloneses ${ }^{40}$. Destacar, la posibilidad de comerciar con cristianos a crédito, que aún con limitaciones ofrece un abanico diverso entre los que destacarian: vestidos, maíz, cereales, aceite, canem, lino, azafran, y otros artículos; del mismo modo, se aborda la imposibilidad de alargar las deudas contraídas y algunos derechos judiciales ${ }^{41}$. Las nuevas medidas, conforman un conjunto de cambios, ofreciéndonos una regulación en asuntos en los que los judíos parecen ser parte importante. Cuarenta años después de la conquista cristiana, algunos grupos judáicos, están en posesión de bienes inmuebles y derechos que les conceden un grado de importancia en el conjunto de la sociedad insular y supraregional ${ }^{42}$.

El 25 agosto 1273, el monarca confirma los privilegios otorgados a los judíos mallorquines mediante la siguiente apreciación: «confirmat privilegia judeorum, et emptionem domorum eorum et potestatem emendi in futurum. Cristianus non habitet cum judeo. Concedit etiam quod judei possit decollare in macello cristianorum ${ }^{43}$. En la misma década, y hasta los años ochenta del mismo siglo, se proce-

38 ARM, ECR. 643, 349, 350 y 16. Citados en: Soto COMPANY. Ricard: “Alguns homes rics: el paper dels jueus de Mallorca durant el segle XIII". Butlleti de la Societat Catalana d'Estudis Historics [Barcelona], 7 (1996), pp. 27-39.

39 Ver las actividades resumidas en la Tabla l.

40 REgne, Jean: "Catalogne des actes de Jaime 1er, Pedro 111 et Alfonso IIl, concernant les juits (1213-1291)", Revue des Etudes Juives [Paris], 69 (1919), pp. 2281-2282.

41 En los pleitos entre judios y cristianos, serán necesarios el tesímonio de un judio y de un cristiano, menos en los que atañen prestamos (hacen falta sólo dos cristianos).

4: Son múltiples las actividades que estos realizarán fuera de Mallorca, ver ejemplo: OLLICH ICASTANYIR, Inmaculada: "Aspectes econòmics de l'activitat dels jueus de Vic. segon el Libri ludeorum (1266-1278)", Miscel lània de Textos Medievals [Barcelona], 3 (1985), pp. 3-118.

4a Archivo de la Corona de Aragón, Reg. XIX, Jacobi I, f. 47 
derá a una política - digamos que aperturista - de asentamiento demográfico, la misma podemos observarla desde la cartas de franqueza ${ }^{44}$ otorgadas individualmente a judios, siendo en su mayoría otorgadas por Pere de Caldes.

\section{LA CREACIÓN DEL CALL: MOVIMIENTOS ECONÓMICOS Y ORGANIZACIÓN POLITICO-SOCIAL}

A finales de 1285, con la nueva anexión de Mallorca a la Corona de Aragón, se producen algunos cambios que afectarán directamente a los grupos hebreos instalados. En cierta medida, podríamos afirmar que se aprovecha la nueva coyuntura política para reestructurar algunos de los privilegios que éstos habían adquirido. Del mismo modo, grupos de presión cristianos pidieron y obtuvieron ventajas juridicas, además de reclamar una cierta crítica y derecho sobre el estatus adquirido por los judíos desde su llegada, el cambio del monarca, fue - sin duda - aprovechado para ejercer cierta presión, algunas de estas medidas están encaminadas al reordenamiento urbano, político e institucional de la ciudad ${ }^{45}$. Alfonso el Liberal, lleva a cabo una política, dura y pactista a la vez, muy efectiva: "confirmar la franquicia que facultaba a los pobladores del reino de Mallorca para negociar en territorios de la Corona de Aragón, sin devengar leuda, peatge, pes e mesuratge, y garantía de no imponer en Mallorca leuda nova o peatge" $y$ "salvoconducto a los sarracenos y judíos que negociaran en Mallorca, pagant los drets que son acostumats e deuen èsser pagats.

Entre estas propuestas de Alfonso III de Aragón, están el agrupamiento de la comunidad y su segregación, la imposibilidad de comprar o adquirir bienes inmuebles o rentas, así como la exclusión de ocupar cargos administrativos de cierto relieve ${ }^{46}$. Las nuevas pautas, no son exclusividad del territorio balear, asi observamos como en Barcelona ${ }^{47}$, a finales del siglo XIII, se aprecia un declive de la participación judaica en la política, sus cargos serán ocupados paulatinamente por los nuevos burgueses (básicamente comerciantes) catalanes. Con los cambios,

44 Tabla II. Recordar aqui que existen precedentes de cartas de franqueza otorgadas a judios en territorio de repoblación, ver: SERRANO DAURA, Josep (coor.): Les Cartes de població cristiana i de seguretat de jueus i sarraïns de Tortosa (1148/1149). Actes, Universitat Internacional de Catalunya, Barcelona, 2000.

45 ARM, ECR 349, f. 246. Arnau Burgues, batle de Mallorca, compra -en nombre del Rey- casas de la familia Mocatil en la zona de Almudaina. Sobre la política del nuevo reinado, ver: SANTAMARIA ARANDEZ, Álvaro: "La politica municipal de Alfonso el Liberal en el Reino de Mallorca (1285-1291)", La ciudad Hispánica durante los siglos xill al xvi, II, Universidad Complutense, Madrid, 1985, pp. 1271-1299.

46 Archivo del Reino de Mallorca, Rosselló Vell, f. 170 v. Ver asimismo: Quadpado, José Maria: Privilegios y Franquicias de Mallorca, Palma de Mallorca, 1895.

47 BAEF, Yitzhak: Toledot ha-yehudim bi-Sefarad ha-nostrit, Am Oved Ltd. Publishers, Tel Aviv, 1945 (edición castellana: Historia de los judios en la España cristiana, Riopiedras, Barcelona, 1998); AsSIS, Yom Tov: "La participación de los judios en la vida económica de Barcelona, s. XIII-X!v", Jornades d'Història dels Jueus a Catalunya, Ajuntament de Girona, Gerona, 1990, pp 77-92; y, SOBREQUES I CALLICO, Jaume; Rovira, M.: “Prèctecs jueus a la ciutat de Barcelona en el segle XIII (1274-1294)", Miquel Coll i Alentorn. Miscel lània d'Homenatge, Barcelona, 1984, pp. 271-289. 
también nos encontraremos con medidas que a simple vista, pueden parecernos contradictorias. El propio monarca aragonés, también dictaminará algunas disposiciones de carácter tolerante. Así, el 28 de agosto de 1286, autoriza a los judíos a poseer carnicería propia ${ }^{48}$, siendo Jocef Coffe el poseedor del derecho cuatro años más tarde de la única que tenemos documentada ${ }^{49}$. La confirmación de Alfonso III, de la ubicación de la nueva aljama data del 21 de diciembre de $1290^{50}$. En la misma, y en torno a una calle principal, se articularán la sinagoga y la carnicería comunal. Ésta estará completamente cerrada, y contará con puertas de acceso ${ }^{51}$. El denominado call nuevo, se establece en un emplazamiento en torno a las posesiones que la Orden del Temple y otros titulares poseen en la zona sudeste de la urbe. Estas operaciones, constatadas documentalmente, ofrecen toda una serie de operaciones de venta y cambio en el ámbito inmobiliario en los alrededores de la Orden y en otros lugares de la ciudad de gran interés, más si cabe al saber que muchos de estos movimientos son anteriores a la aquiescencia de esta nueva ubicación.

Volviendo al nuevo establecimiento de la comunidad judía, a primera vista, parece estar un tanto alejadada de su anterior ubicación. ¿A qué debemos esta ubicación?, si nos atenemos momentaneamente y observamos la progesiva expansión de la ciudad hacia varias zonas, advertimos que los judios segirán estando en una situación que podríamos juzgar como privilegiada ${ }^{52}$. Para ello, señalamos que se trata de un emplazamiento cercano a la ribera del mar, protegida por muros internos y externos; asi la nueva aljama no perderá su relación con las actividades comerciales y artesenales emergentes en la ciudad, en la que esta minoría jugaba un papel más que importante. Estamos pues, antes un proceso de reordenamiento también urbano, donde el monarca aragonés, aprovecha la situación para fortalecer su proyecto político para la entidad balear. ¿Qué es y a qué obedece la creación de la aljama?: Varias han sido las interpretaciones sobre la finalidad, el sentido y el objetivo de la organización judáica en aljamas. En primer lugar, el térrnino designaría - según David Romano ${ }^{53}$ - a la institución jurídico-social que agrupa a dicho colectivo. La problemática, reside en las fechas de creación de las mismas, puesto que si anteriormente existian grupos numéricamente con entidad considerable y suficiente, ¿porqué aparecen las juderías a finales del siglo x\| y principios del xIII?. En el ámbito hispánico, tenemos constancia de la creación en 1170 de la judería de Tudela, siguiéndole cronológicamente las de Zaragoza (1175), Zorita (1212) y Barcelona (1244) entre otras.

48 Regne, Jean: Catalogue..., doc. 1630.

19 Archivo de la Corona de Aragón. Reg. 194, f. 93v

50 Archivo de la Corona de Aragón, Reg. 194, f. 93v

5. Hasta el momento no hemos conservado ningún documento con el número de puertas, ni la ubicación de las mismas, pero si el que refiere a la construcción, rer: Archivo de la Corona de Aragón, Reg. 83 , f. $100-100 \mathrm{v}$.

52 La tolerancia en la política real, y el desarrollo de determinados privilegios, estarían asociados a la entrada de capital y a su permisividad a aquellos grupos que serian capaces de reativar las actividades económicas en determinados territorios de frontera (física o mental). Ver: MOORE, R.I: La formación de una sociedad represora (Poder y disidencia en la Europa Occidental, 950-1250). Critica, Barcelona, 1989.

53 Romano VENTURA, David: "Aljama frente a juderia, call y sus sinónimos", Sefarad [Madrid], XXXIX (1979), pp. 347-354. 
Para el caso mallorquín, la finalidad del agrupamiento, obedece -en nuestra opinión- a garantizar en un primer lugar la pervivencia del coletivo ${ }^{54}$, asi como a propiciar el mantenimiento de lazos y apoyos propios de una determinada entidad ${ }^{55}$. Del mismo modo, consideramos oportuno indicar que desde la monarquia existe una mayor ambición y diversidad que deriva en un control fiscal ${ }^{56}$ o social de determinados grupos, asi como una mayor complejidad organizativa que se encamina hacia la aparición de nuevas corporaciones. Ambición, que en muchas ocasiones puede quedar limitada o retocada ante las presiones y los avances de las nuevas agrupaciones de importancia económica que ahora emergen en las ciudades medievales. Esta presión, conjuntamente con otros motivos, hace que en muchas ocasiones se tomen medidas que se contradicen.

Para facilitar los puntos anteriormente desarrollados, a partir del reinado de Jaime I se articulan una serie de medidas de carácter político, orientadas sin duda a facilitar las nuevas espectativas. En primer lugar, se establece una libertad de movimiento entre los territorios de la Corona de Aragón para la minoría judía, un salvoconducto mediante el cual se reconoce el derecho a moverse con facilidad por los diversos reinos de la confederanción. La medida, genera una gran aceleración no sólo en lo meramente humano, sino también en los que a capital y mercaderias se refiere. También, y debido a las excelentes condiciones geográficocomerciales que ofrece el reino insular, se ofrece a los judios baleares una autonomía fiscal independiente de las demas aljamas. La medida, genera el movimiento de grupos de judios que habian actuado anteriormente en diversos territorios del dominio real.

En la capital balear, se establecerán grandes mercaderes cuya actuación podemos situarla en un hinterland de carácter supra-local y pequeños artesanos encargados de talleres de iniciativa local, a la suma los predominantes en núme$\mathrm{ro}^{57}$. Se trataría de un universo de situaciones muy complejas y realmente poco conocidas, cuya mejor interpretación se realizará a medida que avanza el conocimiento de nuevas fuentes. En esta situación, los negocios más característicos estarían relacionados con el enérgico y emergente sector textil balear.

La creación de la Corona de Mallorca, con la incorporación de los territorios peninsulares pirenáicos favorece la introducción de mercantes perpiñaneses en el

5 CANTERa Montenegro, Enrique: Aspectos de la vida cotidiana de los judios en la España medieval, Universidad Nacional de Educación a Distancia, Madrid, 1998.

55 LACAVE, José Luis: Juderias y Sinagogas Españolas, Editorial Mapfre, Madrid, 1992.

56. Sobre las transformaciones de la hacienda y la fiscalidad real durante la segunda mitad del siglo Xlit, ver: LADERO QUESADA, Miguel Ángel: "Las transformaciones de la fiscalidad regia castellano-leonesa en la segunda mitad del siglo XII! (1252-1312)". Historia de la Hacienda española (épocas antigua y medieval). Homenaje al profesor Garcia de Valdeavellano, Instituto de Estudios Fiscales, Madrid, 1982. pp. 319-406. Para territorios castellanos, Laredo señala una mayor complejidad y reorganización encaminada hacia una mejor efectividad

Iradiel Murugarren. Paulino: "Metrópolis y hombres de negocios (siglos XIV y XV)", XXIX Semana de Estudios Medievales de Estella 'Las Sociedades Urbanas en la España Medieval', Gobierno de Navarra, Pamplona, 2003, pp. 277-310. 
mediterráneo occidental ${ }^{58}$, a partir de estos momentos proliferan hombres de negocios, recaudadores de rentas y sociedades mercantiles. Jaime II de Mallorca, pretende regular el crédito, las sucesiones y las ventas de mercancías en Perpiñán ${ }^{59}$, ciudad económicamente muy dinánima en la que se han establecido cualificados hebreos de origen franco ${ }^{60}$, participando éstos de forma específica en la comercialización y redistribución de productos ${ }^{61}$. 1298, Jaime II, recupera el gobierno del reino y revoca algunas ordenes anteriores, entre ellas, la prohibición de poseer bienes inmuebles. Produciéndose así, una problemática con la venta de las propiedades fuera del call asi como las de la nueva ubicación.

Durante la etapa de infante, Jaume II de Mallorca adquiere la suficiente experiencia ${ }^{62}$ que hace de su reinado una etapa de expansión. En este sentido, existe una politica patrimonial ${ }^{63}$ por parte de la monarquía que posee algunos precedentes, aunque será Jaime II el artífice de un proceso encaminado a la ampliación patrimonional, así como a la reoganización de la hacienda y una mayor efectividad fiscal. Para ello, encaminará muchas de sus medidas hacia el colectivo templario. Éstas medidas, podriamos encuadrarlas, dentro de un proceso general sobre la Orden, que a nivel quasi europeo, desemboca en la convocatoria del Concilio de Viena (13111312) por parte de Clemente $V$, para tratar la abolición de la misma. Las disposiciones sobre los templarios, afectarán de manera directa e indirecta beneficiosamente al patrimorio real. Los bienes serán confiados al obispado de Mallorca, mientras que sus antiguos propietarios se enfrentarán a un proceso sumarísimo de confiscación

$s 8$ RiERA I MELIS, Antoni: «Perpiñán, 1025-1285. Crecimiento económico, diversificación social y expansión urbana", ABULAFIA, David; GARi, Blanca (dir.): En las costas del Mediterráneo Occidental. Las ciudades de la Península Ibérica y del reino de Mallorca y el comercio mediterráneo en la Edad Media, Omega. Barcelona, 1996, pp. 1-61.

Archives Comunales de Perpignan. Livre Vert Majeur, f. 60r-66r. Citado en: RIERA I MELIS, Antoni: "Perpiñán, 1025-1285. Crecimiento económico... p 30, ver también: Assis, Yom Tov: “Les juifs de Montpellier sous la domination aragonaise", Revue des Études Juives [Paris], CXLVIII (1989), pp. 5-16; y KaTSURA, Hideyuki: "L'administration financière de la seigneurie de Montpellier sous le règne de Jacques $l^{* \prime \prime}$ le Conquèrant", Sanchez Martinez, Manuel (ed.): Fiscalidad real y finanzas urbanas en la Cataluña Medieval, Consejo Superior de Investigaciones Cientificas, Barcelona, 1999, pp. 13-53.

60. Asi por ejemplo, el judio marsellés, Bonisaac Ferrusol, actúa como representante de algunos comerciantes que circular por Mallorca hacia finales de 1248. Sobre las actividades que los judios francos desarrollan, es imprescindible consultar: EMERY, Richard Wilder: The jews of Perpignan in the thirteenth century: An Economic Study Based on Notarial Records, Columbia University Press, New York, 1959; LOEB, 1.: «Les négociants juifs à Marseille au milieu du XIIle siècle», Revue des Etudes Juives [Paris], XVI (1888), pp. 73-83: Cremieux, A.: "Les juifs de Marseilles au Moyen Âge", Revue d'Etudes Juives [Paris], 46 (1903), pp. 1-36 y 246-268; y, KAHN, S: “Documents inédits sur les juifs de Montpellier", Revue des Études Juives [Paris], XIX (1889), pp. 259-281; "Documents inédits sur les juifs de Montpellier. \|», Revue des Études Juives [Paris], XXII (1891), pp. 265-279. También contamos con interesantes aportaciones sobre el papel y la importancia de los judios que migran: Assis, Yom Tov: "Juifs de France réfugiés en Aragon (XIIle-XIVe siècles)", Revue des Études Juives [Paris], CXLII (1983), pp. 209-227.

61 Garcia Marsilla, J. V.: "Puresa i negoci. El paper dels ueus en la producció i comercialització de queviures a la Corona d'Aragó", Revista d'Història Medieval [Valencia], 4 (1993), pp. 161-182.

6? Cateura Bennassea, Pau: Mallorca en el segle xim. El Tall Editorial, Palma, 1997.

63 Cateura Bennasser. Pau: “Mallorca y la politica patrimonial de la Monarquia (siglos xin y primera mitad del siglo XIV)", Estudis Baleàrics [Palma], 6 (1982), pp. 79-130; Ver tambiér, del mismo autor: "La 'Ciutat de Mallorques' y las villas mallorquinas: presión fiscal y conflictos sociales" V Congreso de Estudios Medievales 'Finanzas y Fiscalidad Municipal', Fundación Sánchez Albornoz, Ávila, 1997, pp. 275-300. 
patrimonial y judicial ${ }^{64}$. Ante la debilidad y dispersión geográfica del reino privativo, comparecen medidas correccionistas ${ }^{65}$ de carácter político, que se encaminan a la mejor vertebración económica de los territorios del mismo.

Recordemos, que debido a la prestación ofrecida por las Órdenes Militares en el proceso de conquista, éstas habian acumulado un patrimonio más que importante en la Isla. Así, no es de extrañar la operación recalificadora en los territorios de la Órden del Temple (por sus notorias posesiones). Pau Cateura advierte, que se trataría de movimientos selectivos encaminados a la dotación de sedes adecuadas para la monarquia (tales como la Almudaina o las residencias secundarias) y a la reorganización de los intramuros ${ }^{66}$ y exteriores de la ciudad. Las medidas podemos considerarlas como ambiciosas y en cierta medida agresivas cuando se trata de embargos de gran importancia. Del mismo modo, el mismo autor en otro trabajo ${ }^{67}$, también nos encamina a pensar que la operación de reubicación de la comunidad hebrea, sería algo costosa y necesariamente llevaría consigo una complicada operación de financiamiento. Pero el coste al que nos referimos, no es únicamente económico, puesto que también acontecen conflictos morales y religiosos. En esta línea, la iglesia de Mallorca ${ }^{68}$, se manifiesta de forma casi unánime contraria a estas nuevas medidas, y confrontarán, conjuntamente con otros grupos nobiliarios, a presionar social y políticamente. José María Quadrado, recordaba con estas palabras, la nueva situación creada en el seno del Reino de Mallorca:

"Con ojos bien distintos de los ciudadanos, miraban los forenses a los judíos... tiene una explicación económica al observar la posición tan distinta que aquellos ricos capitalistas y logreros respecto a la riqueza mercantil» 69 .

Sin duda, este perjudicial y negativo imaginario que se está creando en torno a los judíos está favorecido por las actividades que algunos desarrollan. Ya se ha señalado que la preponderancia del judío como comerciante, era consecuencia lógica de una situación social y religiosa clara ${ }^{70}$ : no podían - sin privilegios- poseer

${ }_{64}$ Ver proceso, Perez Martinez, Lorenzo: “Documentos conservados en los registros vaticanos relativos al cuarto pontificado de Mallorca (D. Guillermo de Vilanova 1304-1318)", Bulleti de la Societat Arqueologica Lur liana [Palma], XXXIII (1970), pp. 228-253.

65 Riera IMElis, Antoni: “Mallorca 1289-1311, un ejemplo de 'planificación económica' en la época de plena expansión", Estudios Históricos y Documentos de los Archivos de Protocolos [Barcelona], V (1977), pp. 199-244.

${ }_{66}$ Cateura Bennasser, Pau: "Mallorca y la politica..., entre las medidas se encuentran: reordenamiento de la zona del muelle, traslado y reagrupamiento judáico y desvio del cauce la Riera.

67 Cateura Bennasser, Pau: "Prejuicio religioso y conflicto social en una pequeña sociedad mediterránea. El caso de Mallorca (1286-1435)", Anuario de Estudios Medievales [Barcelona], 25.1 (1995), pp. 235-253.

i: PONS PASTOR, Antoni: Los judíos del Reino de Mallorca durante los siglos XIIi y XIV, Miquel Font Editor, Palma, 1984, vol II, doc. 22. Para ver el poder de los grupos eclesiásticos ver: LOPEZ BONET, Josep Francesc: "La dotació de l'església de Mallorca desprès de la conquesta cristiana (1229-1280)", XII Congrès d'Histoire de la Couronne d'Aragon, II, Montpellier, 1988, pp. 115-124.

69 Quadrado, José Maria: Forenses y ciudadanos. Historia de las disensiones civiles de Mallorca en el siglo xv. Imprenta Palma, Palma, 1847.

70 LOPEZ, Roberto S.: La revolución comercial en la Europa medieval, El Albir Universal, Barcelona, 1981 
o establecerse en aristocracia de base militar o territorial, com asimismo tampoco podían ejercer una función religiosa digamos que privilegiada. Por tanto, en cierta medida, se verían obligados a ganarse la vida en una profesión usual. Los monarcas aragoneses eran muy conscientes del potencial mercantil ${ }^{71}$, y por ende económico, de Mallorca. Ésta tenía importantes contactos políticos y comerciales con puertos del Atlántico, y del Mediterráneo ${ }^{72}$ y en este sentido, los judíos jugarian un cometido determinante.

Éstos y otros acontecimientos de la centuria, generarán un progresivo cambio mental en determinados grupos de población cristiana, siendo por tanto el punto de partida de los acontecimientos violentos que tendrán lugar a finales de siglo ${ }^{73}$.

11 ABULAFiA, David: "El comercio y el reino de Mallorca, 1150-1450", ABulafia, David:; Gari, Blanca (dir.): En las costas del Mediterráneo Occidental. Las ciudades de la Península lbérica y del reino de Mallorca y el comercio mediterráneo en la Edad Media, Omega, Barcelona, 1996, pp. 115-155; y, ABULAFIA, David: "From privilege to persecution. Crown, church and synagogue in the city of Majorca, 122.91343", en: Church and city. 1000-1500. Essays in Honour of Christopher Brooke. Amsterdam, 1992, pp. $121-126$.

72. Cateura Bennasser, Pau: "Mallorca a la segona meitat del segle XIII (aspectes polítics i financers fins a 1276", Estudis Baleàrics [Palma], 17 (1985), pp. 57-86; "La cuentas de la colonización feudal (Mallorca, 1231-1245)", En la España Medieval [Madrid], 20 (1997), pp. 57-141. Desde un punto de vista fiscal y económico, Pau Cateura analiza las áreas de influencia politicas y los lazos comerciales del reino mallorquín durante el siglo XIII.

73 Consideramos la existencia de progresivos cambios de la mentalidad balear hacia la minoría judia, la misma iniciara una creciente presión que desencadenará en el asalto al barrio judío de 1391, ver mi trabajo: «Apreciaciones sobre la part forana. Mentalidad y marginalidad en el siglo XIV mallorquín (1391)", Mayurqa [Palma], 28 (2002), pp. 241-246. 
TABLA I. OPERACIONES DE VENTA-CAMBIO. DE JUDIOOS A CRISTIANOS $(1285-1298)^{74}$

\begin{tabular}{|c|c|c|c|c|c|}
\hline Fecha & $\mathrm{De}$ & A & Operación & Zona & Detalle \\
\hline $\begin{array}{l}1285 \\
3 \text { junio }\end{array}$ & $\begin{array}{l}\text { Isaac, fill de } \\
\text { Abraham } \\
\text { Benhacdach }\end{array}$ & Pere Tintorer & $\begin{array}{l}\text { Venta } \\
\text { (30 libras) }\end{array}$ & Casas & $\begin{array}{l}\text { Dentro de la } \\
\text { Ciudad }\end{array}$ \\
\hline $\begin{array}{l}1287 \\
3 \text { marzo }\end{array}$ & $\begin{array}{l}\text { Jucef fill de } \\
\text { Mossé } \\
\text { Abenelfagg }\end{array}$ & $\begin{array}{l}\text { Ferrer des } \\
\text { Colomers }\end{array}$ & $\begin{array}{l}\text { Venta } \\
\text { (30 libras) }\end{array}$ & Casas & $\begin{array}{l}\text { Cerca de la } \\
\text { carnicería de } \\
\text { Nuno Sanç }\end{array}$ \\
\hline $\begin{array}{l}1291 \\
13 \text { julio }\end{array}$ & $\begin{array}{l}\text { Isaac } \\
\text { Benfaedal y } \\
\text { mujer }\end{array}$ & $\begin{array}{l}\text { Pere } \\
\text { Vilabertran }\end{array}$ & Cambio & $\begin{array}{l}\text { Monestir de } \\
\text { Santa } \\
\text { Margalida }\end{array}$ & $\begin{array}{l}\text { Albergue en la } \\
\text { calle Guillem } \\
\text { Vilar }\end{array}$ \\
\hline $\begin{array}{l}1291, \\
19 \text { julio }^{78}\end{array}$ & $\begin{array}{l}\text { Bolax ben } \\
\text { Maymó, } \\
\text { Jacob ben } \\
\text { Maimó et alii. }\end{array}$ & $\begin{array}{l}\text { Flor (viuda de } \\
\text { Ramon Ferrer) }\end{array}$ & Cambio & $\begin{array}{l}\text { Monestir de } \\
\text { Santa } \\
\text { Margalida }\end{array}$ & $\begin{array}{l}\text { Albergue en el } \\
\text { call }\end{array}$ \\
\hline $\begin{array}{l}1291 \\
3 \text { agosto }^{79}\end{array}$ & $\begin{array}{l}\text { Maymó } \\
\text { Abennono y } \\
\text { mujer }\end{array}$ & $\begin{array}{l}\text { Guillem des } \\
\text { Vilar }\end{array}$ & $\begin{array}{l}\text { Venta } \\
\text { (50 libras) }\end{array}$ & $\begin{array}{l}\text { Monestir de } \\
\text { Santa } \\
\text { Margalida }\end{array}$ & \\
\hline $\begin{array}{l}1291 \\
13 \text { agosto }\end{array}$ & $\begin{array}{l}\text { Abrafim ben } \\
\text { Marguit y } \\
\text { mujer }\end{array}$ & Pere Pintener & Cambio & $\begin{array}{l}\text { Albergue } \\
\text { (zona M. de } \\
\text { Santa } \\
\text { Margalida) }\end{array}$ & $\begin{array}{l}\text { Casa situada } \\
\text { en el Temple }\end{array}$ \\
\hline $\begin{array}{l}1291 \\
13 \text { agosto }\end{array}$ & $\begin{array}{l}\text { Jacob Dedici } \\
\text { y mujer }\end{array}$ & $\begin{array}{l}\text { Guerau de } \\
\text { Marina (notari) }\end{array}$ & $\begin{array}{l}\text { Venta } \\
\text { (120 libras) }\end{array}$ & Casas & $\begin{array}{l}\text { Costa del forn } \\
\text { de n'Escala }\end{array}$ \\
\hline $\begin{array}{l}1292 \\
11 \text { febrero }^{82}\end{array}$ & $\begin{array}{l}\text { Isaac ben } \\
\text { Fardal y mujer }\end{array}$ & $\begin{array}{l}\text { Arnau de } \\
\text { Castello }\end{array}$ & $\begin{array}{l}\text { Venta } \\
\text { (225 libras) }\end{array}$ & $\begin{array}{l}\text { Monestir de } \\
\text { Santa } \\
\text { Margalida }\end{array}$ & \\
\hline $\begin{array}{l}1292 \\
4 \text { marzo }^{83}\end{array}$ & $\begin{array}{l}\text { Vidal Cohén y } \\
\text { mujer }\end{array}$ & $\begin{array}{l}\text { Guido Valle de } \\
\text { Ter }\end{array}$ & $\begin{array}{l}\text { Venta } \\
\text { (120 libras) }\end{array}$ & Casas & $\begin{array}{l}\text { Dentro de la } \\
\text { Ciudad }\end{array}$ \\
\hline $\begin{array}{l}1292 \\
24 \text { diciembre }^{84}\end{array}$ & $\begin{array}{l}\text { Jucef Levi y } \\
\text { mujer }\end{array}$ & Pere de Clarà & $\begin{array}{l}\text { Venta } \\
\text { (53 libras) }\end{array}$ & Casas & $\begin{array}{l}\text { Dentro de la } \\
\text { Ciudad }\end{array}$ \\
\hline
\end{tabular}

${ }^{74}$ Los documentos de esta tabla han sido regestados por Ramón Rosselló Vaquer (La Ciutat de Mallorca després de la conquista de 1229 (Documentari 1230-1300). Edicions Roig i Montserrat, Palma, 2004.
ARM, ECR. 351, f. 46 .
ARM, ECR. 351, f. $167 \mathrm{~V}$
ARM, ECR. 352, f. $214 \mathrm{~V}$
ARM, ECR. 352, f. $216 \mathrm{v}$
ARM, ECR. 352, f. $219 \mathrm{v}$.
ARM. ECR. 352, f. 221v.
ARM, ECR. 352, f. 223.
ARM ECR. 352, f. $243 \mathrm{v}$
ARM, ECR. 352, f. 247V
RA ARM, ECR. 353, f. $73 \mathrm{v}$. 
Política, economía y fiscalización de un espacio urbano. El establecimiento de la Aljama...

TABLA I. (continuación)

\begin{tabular}{|c|c|c|c|c|c|}
\hline Fecha & $\mathrm{De}$ & A & Operación & Zona & Detalle \\
\hline $\begin{array}{l}1293 \\
21 \text { julio }\end{array}$ & $\begin{array}{l}\text { Jucef Moani y } \\
\text { mujer }\end{array}$ & $\begin{array}{l}\text { Asday Bondia } \\
\text { (judio) }\end{array}$ & $\begin{array}{l}\text { Venta } \\
\text { (90 libras) }\end{array}$ & $\begin{array}{l}\text { Mitad de un } \\
\text { albergue en } \\
\text { el call antiguo }\end{array}$ & \\
\hline $\begin{array}{l}1294 \\
5 \text { abrit }\end{array}$ & Abrahim fill & $\begin{array}{l}\text { Guillem de } \\
\text { Cantarelles }\end{array}$ & $\begin{array}{l}\text { Venta } \\
\text { (57 libras) }\end{array}$ & Casas & $\begin{array}{l}\text { Dentro de la } \\
\text { Ciudad }\end{array}$ \\
\hline $\begin{array}{l}1295 \\
16 \\
\text { noviembre } 87\end{array}$ & $\begin{array}{l}\text { Samuel } \\
\text { Addenden }\end{array}$ & $\begin{array}{l}\text { Arnau de } \\
\text { Castelló }\end{array}$ & $\begin{array}{l}\text { Venta } \\
\text { (75 libras) }\end{array}$ & $\begin{array}{l}\text { Cerca de la } \\
\text { capilla }\end{array}$ & \\
\hline $\begin{array}{l}1295, \\
20 \\
\text { diciembre }\end{array}$ & $\begin{array}{l}\text { Jacob ben } \\
\text { Jucef 'Didici' }\end{array}$ & Pere Nicolau & $\begin{array}{l}\text { Venta } \\
\text { (200 libras) }\end{array}$ & Albergue & En la Ciudad \\
\hline $\begin{array}{l}1296 \\
2 \text { marzo }^{89}\end{array}$ & $\begin{array}{l}\text { Cacim Moxat } \\
\text { hijo de } \\
\text { Salamó }\end{array}$ & $\begin{array}{l}\text { Ramon } \\
\text { Muntaner } \\
\text { (mercader) }\end{array}$ & $\begin{array}{l}\text { Venta } \\
\text { (50 libras) }\end{array}$ & Casas & $\begin{array}{l}\text { Costa de Arnau } \\
\text { Benet }\end{array}$ \\
\hline $\begin{array}{l}1296 \\
12 \text { junio }\end{array}$ & Jucef Leví & $\begin{array}{l}\text { Maria viuda de } \\
\text { Arnau de } \\
\text { Bellpuig }\end{array}$ & $\begin{array}{l}\text { Venta } \\
\text { (128,30 libras) }\end{array}$ & Casas & $\begin{array}{l}\text { Calle de Sant } \\
\text { Bartomeu }\end{array}$ \\
\hline $\begin{array}{l}1297 \\
30 \text { enero }\end{array}$ & $\begin{array}{l}\text { Jucef Ses } \\
\text { Portes }\end{array}$ & $\begin{array}{l}\text { Ugolino de } \\
\text { Bisanyo } \\
\text { (genoves) }\end{array}$ & $\begin{array}{l}\text { Venta } \\
\text { (35 libras) }\end{array}$ & Albergue & $\begin{array}{l}\text { Antiguamente } \\
\text { era una } \\
\text { adoberia }\end{array}$ \\
\hline $\begin{array}{l}1298, \\
5 \text { diciembre } 92\end{array}$ & Asday Bondia & $\begin{array}{l}\text { Guillem } \\
\text { Valenti }\end{array}$ & $\begin{array}{l}\text { Venta } \\
\text { (70 libras) }\end{array}$ & Casas & $\begin{array}{l}\text { Cerca de la } \\
\text { capilla de Sant } \\
\text { Bartomeu }\end{array}$ \\
\hline
\end{tabular}

8: ARM, ECR. 353, f. 114.

Bt ARM, ECR. 353, f. 142v.

87 ARM, ECR. 354, f. 69.

88 ARM, ECR. 354, f. 74.

89 ARM, ECR. 354, 1. 86v.

50 ARM, ECR. 354. f. 99.

41 ARM, ECR. 354, 1. 148.

\% ARM, ECR. 355, f. $80 \mathrm{v}$. 
TABLA II. CARTAS DE FRANQUEZA (1263-1283) ${ }^{93}$

\begin{tabular}{|c|c|c|}
\hline Fecha & Nombre & Testimonios \\
\hline 1263, 2 julio & Abraham Atzegardo & \\
\hline 1263, 2 julio & Mossé fill Dechelfalla & \\
\hline 1264,12 enero & Jucef Abramun & \\
\hline 1264,19 febrero & Aissach Ambran & \\
\hline 1265,28 julio & Saat fill de Jecel & $\begin{array}{l}\text { Homar hijo de Jucef, Homan Aben } \\
\text { Agnus, Isaac Cohén y Jaume Mercer }\end{array}$ \\
\hline 1265,30 agosto & Salamó hijo de Aeli & \\
\hline 1265,30 agosto & Abraim Atzagando & \\
\hline 1265,30 agosto & Abraim Abenainus & \\
\hline 1265.30 agosto & Phayon Abenfanyo & \\
\hline 1265,15 octubre & Salamó fill de Abraim Abenainuz & \\
\hline 1265,15 octubre & Abraim Abenainus Almostalf & \\
\hline 1265,15 octubre & Haibo ben Maimó & \\
\hline 1267,16 febrero & Phayon hijo de Samuel ben Bolax & \\
\hline 1267, 8 julio & Mossé hijo de Jaffia Anatjar & \\
\hline 1267,27 julio & Issac hijo de Phayon & \\
\hline 1267,22 agosto & Phayon Cohén & \\
\hline 1267,22 agosto & Jucef Bendaanan & \\
\hline 1268,31 julio & Mussa hijo de Jucef Abenaadde & \\
\hline 1268,10 septiem. & Abrafim hijo de Jucef & \\
\hline 1268,21 octubre & Haaron hijo de Abrafim Chozén & \\
\hline 1269,11 febrero & Salamó hijo de Jucef Abenjacr & \\
\hline 1269,11 febrero & Abrafim aben Jucef Abendauch & \\
\hline 1269, 28 noviemb. & Abrafim hijo de Issac Benmoregit & \\
\hline 1269,28 noviemb. & Jucef Abenabraim & \\
\hline 1272,14 mayo & Salamó fill de Boorach & \\
\hline 1273,29 agosto & Alell fill de Merratxi & Secretarios de la aljama \\
\hline
\end{tabular}

93 ARM, ECR. 348, 349, 350, 643. Los documentos de esta tabla han sido regestados por Ramón Rosselló Vaquer (La Ciutat de Mallorca després de la conquista de 1229 (Documentari 1230-1300), Edicions Roig i Montserrat, Palma, 2004. 
Politica, economia y fiscalización de un espacio urbano. El establecimiento de la Aljama..

\section{TABLA II. (continuación)}

\begin{tabular}{|c|c|c|}
\hline Fecha & Nombre & Testimonios \\
\hline 1273,29 agosto & Mandil ben Abram & Secretarios de la aljama \\
\hline 1273,29 agosto & Phalto Macluf & \\
\hline 1273,30 septiemb. & Vidal Alcani & \\
\hline 1273,2 noviembre & Bensoloma & \\
\hline 1273,2 noviembre & Homer fill de Jacob ben Adde & \\
\hline 1273,4 noviembre & Phixon ben Farroch & $\begin{array}{l}\text { Bonisac de Marsella y Abrafim } \\
\text { Alatzarac (secretarios de la aljama) }\end{array}$ \\
\hline 1278,10 enero & Salamó Cohén & Secretarios de la aljama \\
\hline 1278,20 octubre & Sallom Benmenexi & \\
\hline 1279,3 marzo & Jucef fill de Abrafim Alanzar & $\begin{array}{l}\text { Castello Sardina (jurista), Maani hijo de } \\
\text { Illel, Jucef Ben Phanin, Homer Ben } \\
\text { Phanum (judios secretarios) }\end{array}$ \\
\hline 1280,16 marzo & Jacob ben Salamon & Secretarios de la aljama \\
\hline 1280,17 de mayo & Salamó fill de Jucef Abenaminus & Secretarios de la aljama \\
\hline 1280,17 mayo & Abraym, fill de Aarón Cohén & \\
\hline 1280,4 junio & Isaac ben Jucef Benquabax & \\
\hline 1280,28 junio & Isaac ben Mussa & Secretarios de la aljama \\
\hline 1280,22 septiemb. & Ambram fill de Isaac Abnattauhil & \\
\hline 1280,16 octubre & Abdallac, fill de Abrafim & \\
\hline 1280,31 octubre & Abraym Benarosa & \\
\hline 1281,3 noviemb. & Jacob Abenallel Abennono & $\begin{array}{l}\text { Isaac ben Ambran, Jucef Benaniinus } \\
\text { (secretarios) }\end{array}$ \\
\hline 1282,2 julio & Maimó Tangi & Secretarios de la aljama \\
\hline 1282,17 noviemb. & Macluf Benjucef & \\
\hline 1282,17 noviemb. & Samuel Bajat & \\
\hline 1283,15 febrero & Macluf Benfara & \\
\hline 1283,22 febrero & Mussa Abenifra & \\
\hline 1283,5 marzo & Suleimen Benphayon & \\
\hline 1283,5 marzo & Çayt fill de Jueces & \\
\hline 1283,5 marzo & Davet fill de Salamó & \\
\hline
\end{tabular}

